ABSTRACT 


\section{An Investigation on Non-Invasive Brain-Computer Interfaces: Emotiv Epoc+ Neuroheadset and Its Effectiveness}

\author{
Md Jobair Hossain Faruk \\ Department of Software Engineering \\ Kennesaw State University \\ Marietta, USA \\ mhossa21@students.kennesaw.edu
}

\author{
Maria Valero \\ Department of Information Technology \\ Kennesaw State University \\ Marietta, USA \\ mvalero2@kennesaw.edu
}

\author{
Hossain Shahriar \\ Department of Information Technology \\ Kennesaw State University \\ Marietta, USA \\ hshahria@kennesaw.edu
}

Keywords: Neurotechnology, Brain-Computer Interfaces, Brain-Machine Interfaces, Electroencephalography, Brain.

Neurotechnology describes as one of the focal points of today's research around the domain of Brain-Computer Interfaces (BCI). The primary attempts of $\mathrm{BCI}$ research are to decoding human speech from brain signals and controlling neuro-psychological patterns that would benefit people suffering from neurological disorders. In this study, we illustrate the progress of $\mathrm{BCI}$ research and present scores of unveiled contemporary approaches. First, we explore a decoding natural speech approach that is designed to decode human speech directly from the human brain onto a digital screen introduced by Facebook Reality Lab and University of California San Francisco. Then, we study a recently presented visionary project to control the human brain using BrainMachine Interfaces (BMI) approach. We also investigate well-known electroencephalography (EEG) based Emotiv Epoc+ Neuroheadset and present experimental studies to identify six emotional parameters using brain signals by experimenting the neuroheadset among three human subjects where we utilize two classifiers, Naïve Bayes and Linear Regression to show the accuracy and competency of the Epoc+ device and its associated applications in neurotechnological research. The demonstration indicates $69 \%$ and $62 \%$ improved accuracy for the aforementioned classifiers respectively in reading the performance matrices of the participants. We envision that non-invasive, insertable, and low-cost $\mathrm{BCI}$ approaches shall be the focal point for not 
only an alternative for patients with physical paralysis but also brain understanding that would pave us to access and control the memories and brain somewhere very near. 